\title{
A Proposed Method for Feature Selection using a Binary Particle Swarm Optimization Algorithm and Mutual Information Technique
}

\author{
Mustafa Ayham Abed Alhafedh \\ mouhamed_milan@yahoo.com
}

Omar Saber Qasim

College of Computer Science and Mathematics

University of Mosul, Mosul, Iraq

Received: 07/04/2019

Accepted: 30/06/2019

\begin{abstract}
Feature selection is one of the most important issues in improving the data classification process. It greatly influences the accuracy of the classification. There are many evolutionary algorithms used for this purpose, such as the Particle Swarm Optimization (PSO) in discrete space through the Binary PSO concept. The BPSO optimization algorithm derives its mechanism from the default PSO algorithm but in discrete space. In this research, a hybrid approach was proposed between the BPSO algorithm and Mutual Information (MI) to obtain subsets of features through two basic phases: the first is to use the BPSO algorithm to determine the features affecting the data classification process by relying on an objective function. In the second phase, the MI method is used to reduce the number of features identified by the BPSO method. The results of the proposed algorithm have demonstrated efficiency and effectiveness by obtaining higher classification accuracy and using fewer features than default methods.
\end{abstract}

Keywords: Feature selection; particle swarm optimization; Mutual information technique; classification.

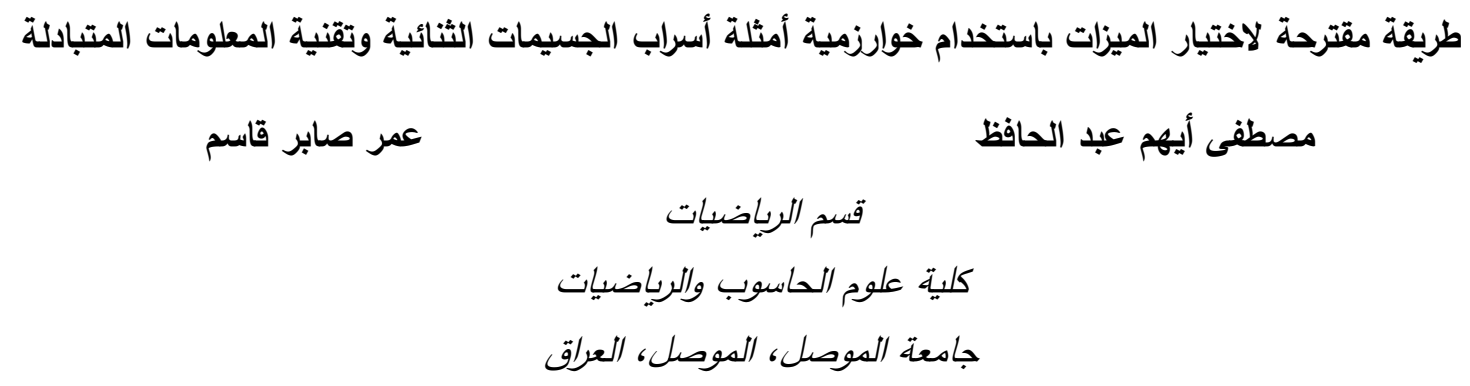

الملخص

تعد مسألة اختيار الميزات Feature selection من أهم المسائل المستخدمة في تحسين عملية تصنيف

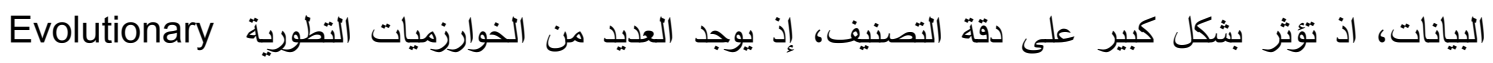

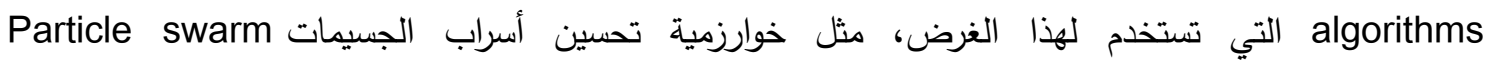
optimization (PSO) خلال مفهوم Binary PSO، إذ تستمد خوارزمية تحسين سرب الجسيمات الثنائية BPSO آلية عملها من خوارزمية الاعتيادية ولكن في مساحة بحث منقطعة ثنائية. اقترحت في هذا البحث طريقة مهجنة بين خوارزمية BPSO

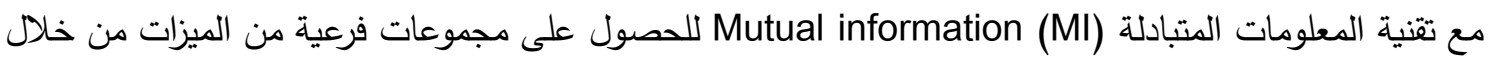


مرحلتين أساسيتين: الأولى هي استخدام خوارزمية BPSO لتحديد الميزات المؤثرة في عملية التصنيف للبيانات بالاعتماد على دالة هدف Objective function مقترحة ، في المرحلة الثانية، تستخدم طريقة المعلومات المتبادلة

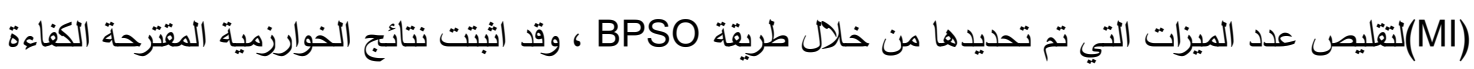
والفعالية من خلال الحصول على دقة تصنيف أعلى و استخدام ميزات قليلة مقارنة بالطرائق الافتراضية الاعتيادية. الكلمات المفتاحية: اختيار الميزة، تحسين سرب الجسيمات، تقنية المعلومات المتبادلة، التصنيف.

Introduction المقدمة

أجريت في السنوات الأخيرة العديد من الدراسات التي تعتمد على محاكاة الأنظمة الذكية الطبيعية Normal intelligent systems ومنها ما يسمى بذكاء السرب Swarm intelligence

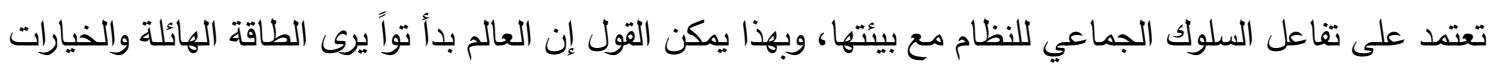

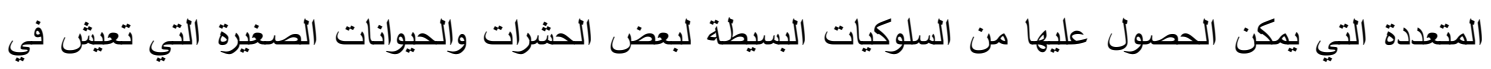

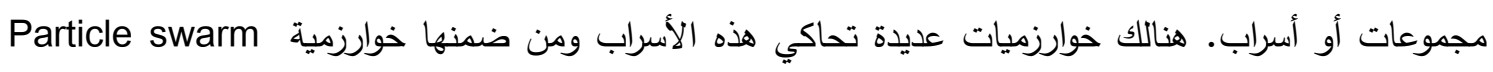
Keptimization (PSO)

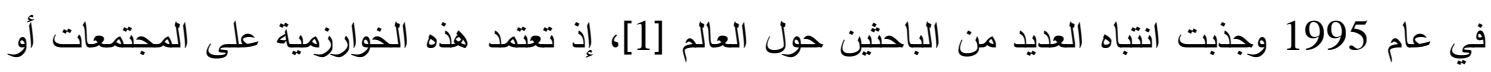
الأسراب Swarm التي شكلت بواسطة حلول عشوائية تسمى الجسيمات Particles ، اذ يرتبط كل جسيم في هذه هن

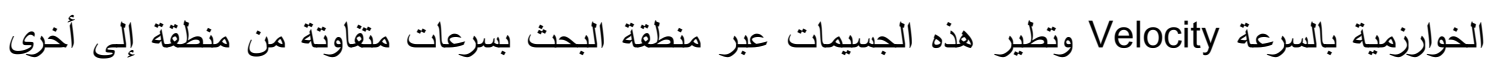

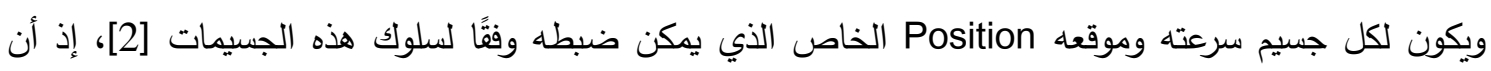

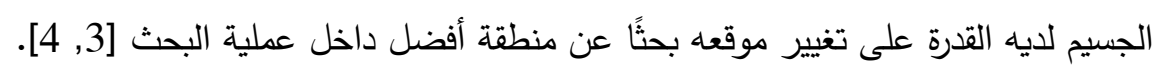
في العام 1997، قام العالمان Eberhardt و Kennedy بتطوير خوارزمية PSO الأساسية، بتحويل خوارزمية

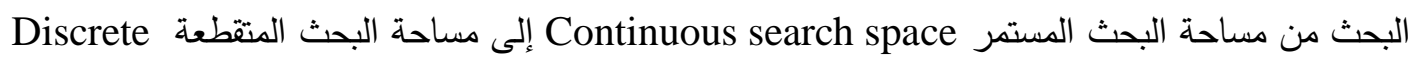
PSO المعدلة على مناطق البحث ثنائية الأبعاد Search space وتسمى الخوارزمية المعدلة الخوارزمية الثنائية BPSO) Binary particle swarm optimization)، إذ إذ تمثيل الجسيمات في الفضاء الثنائي ويمكن أن تأخذ مواقع هذه الجسيمات قيمة ثنائية من (0,1) [6].

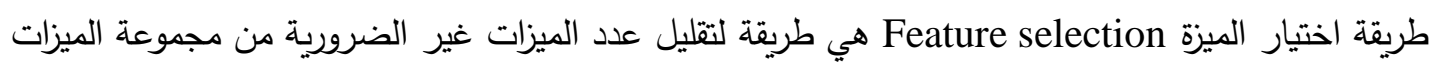

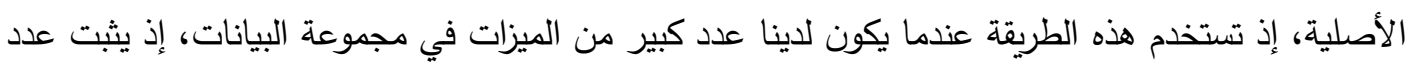
محدد من الميزات الضرورية لتحسين دقة التصنيف [7]. عادة ما تستخدم طريقتان أساسيتان في تحديد الميزة:

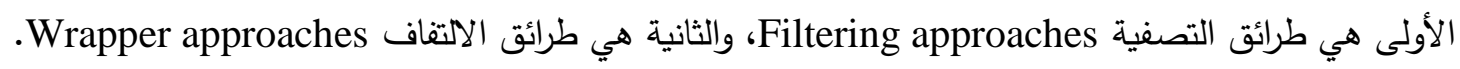

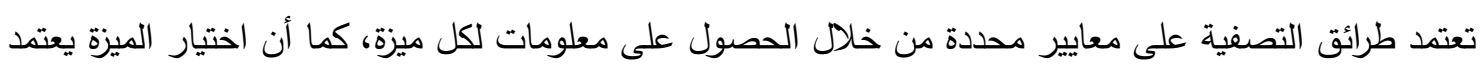
على أداء خوارزمية التصنيف [8]. المعلومات المتبادلة (MI) هي إحدى طرائق التصفية التي يتم حسابها بين متغيرين عشوائيين منفصلين باستخدام الانتروبيا Entropy، إذ يقيس الإنتروبيا للمتغير العشوائي متوسط كمية التئية

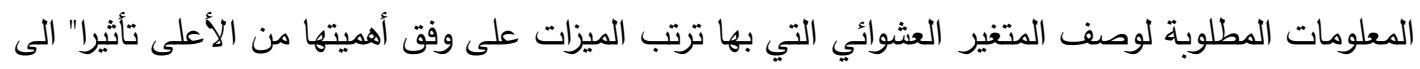
الأقل تأثيرا" في عملية التصنيف [9]. 
2. - 2. - 2. - الاعمال السابقة

يتم في هذه الفقرة التركيز على أهم تفاصيل الأعمال البحثية المختلفة المتعلقة بهذه الدراسة ومن ضمنها

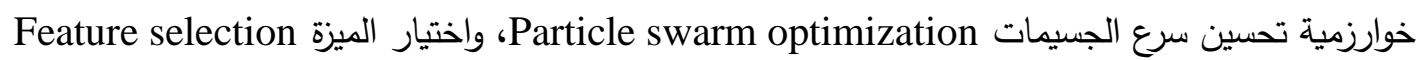
ونستعرضها على النحو الآتي:

فقد قام الباحثان Dichard LBankert David W. Aha. في العام 1993 باستخدام خوارزمية اختيار

الميزة أدارة" للتنبؤ بالطقس مثل الضبان الضباب والاضطرابات الجوية، وحركة العواصف المدارية.

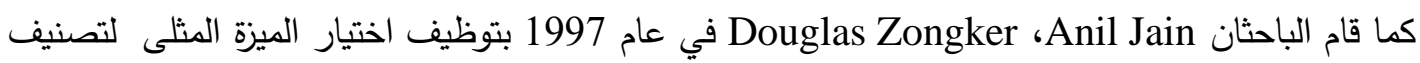
الأراضي على أساس صور الأقمار الصناعية SAR باستخدام أربعة نماذج نسيج مختلفة التي توصف بكونهام ميزات تجميع مستمدة من نماذج نسيج مختلفة، تليها ميزة اختيار النتائج في تحسين دقة التصنيف.

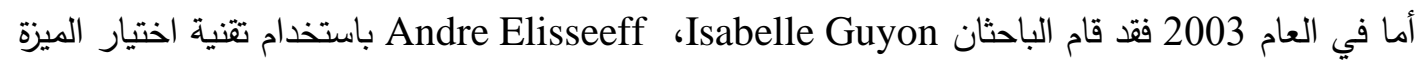
لوصف متغير Variable معين يستخدم في معالجة النصوص من مجموعة متغيرات.

كما عمد الباحثان DW van der Merwe ، تحسين سرب الجسيمات PSO لاختيار البيانات والحصول على دقة عالية في التصنيف.

Pablo A. Estevez, , Michel Tesmer, Claudio A. Perez, Jacek M. أيضا" في 2009 قام الباحثون كurada

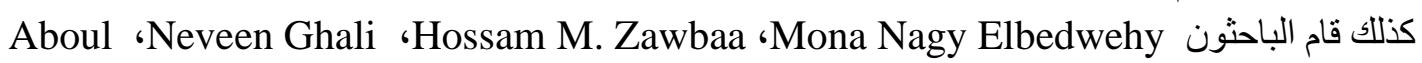
Ella Hassanien باستخدام خوارزمية تحسين سرب الجسيمات الثنائية Binary particle swarm optimization (BPSO) كما قام الباحثان Jane Labadin ،Muhammad Aliyu Sulaiman في عام 2015 باستخدام خوارزمية اختيار الميزة على أساس المعلومات المتبادلة للتتبؤ بالتعلم الآلي.

وفي عام 2018 قام الباحثون Maned ،K.S. Savita ،Manzoor Ahmed Hashmani ،Maryam Zaffar بدراسة خوارزميات اختيار الميزة للتتبؤ بالأداء الأكاديمي كما قدموا تحليلا" لأداء خوارزميات اختيار ميزة الفلتر وخوارزميات التصنيف في مجموعتي بيانات مختلفتين.

3. تحسين سرع الجسيمات Particle swarm optimization

Fish من الخوارزميات التي تحاكي سلوك المجموعات مثل مجموعات الأسماك والحشرات Insects والطيور Birds التي تطير بحثًا عن الطعام بالتعاون بين أعضاء المجموعة، اذ طبق هذه الخوارزمية العالمان Eberhard Kennedy في العام 1995، إذ تعتمد على مجموعة من القيم العشوائية التي لهي

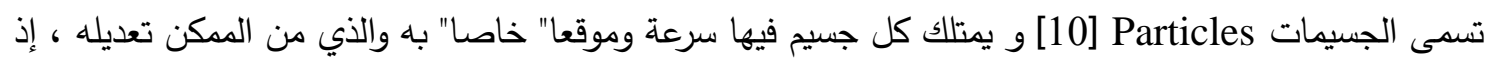
تتحرك هذه الجسيمات داخل مساحة البحث العشوائي [1]. توصف المعادلات الأساسية للسرعة والموقع على النحو $\mathrm{v}_{\mathrm{id}}=\mathrm{v}_{\mathrm{id}}+\mathrm{c} 1 \operatorname{Rand}()\left(\mathrm{p}_{\mathrm{id}}-\mathrm{x}_{\mathrm{id}}\right)+\mathrm{c} 2 \operatorname{Rand}()\left(\mathrm{p}_{\mathrm{gd}}-\mathrm{x}_{\mathrm{id}}\right)$

$\mathrm{x}_{\mathrm{id}}=\mathrm{x}_{\mathrm{id}}+\mathrm{v}_{\mathrm{id}}$

إذ أن $c_{1}$ و هي ثوابت اختيارية موجبة ، وإن Rand() و Rand() هما دالتين عشوائيتين في مدى [0,1) 


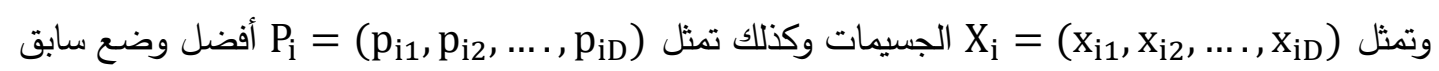

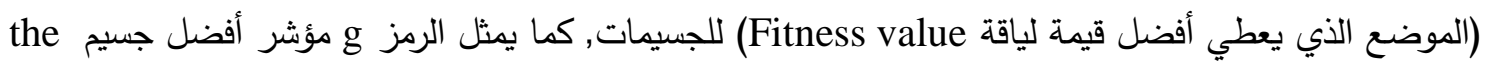
معدل V V V

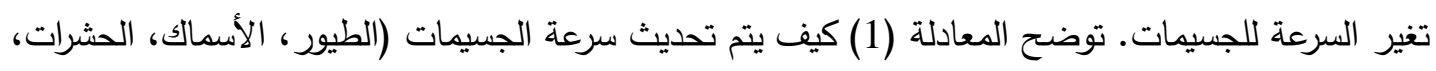
إلخ) بشكل حيوي وأن المعادلة (2) تصف كيفية تحديث الموقع للجسيمات [11]، إذ تتكون المعادلة (1) من ثلاثة أقسام: 1. يحدد القسم الأول أنه لا يمكن تغيير السرعة فجأة. 2. القسم الثاني هو القسم المعرفي الذي يمثل تفكير الجسيم نفسه في تجربة الطيران. 3. القسم الثالث هو القسم الاجتماعي الذي يمثل التعاون بين الجسيمات.

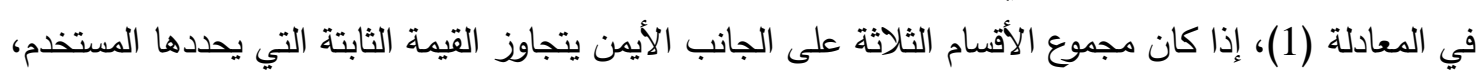

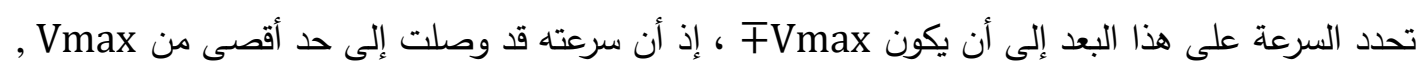

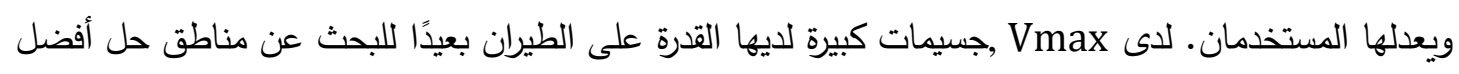

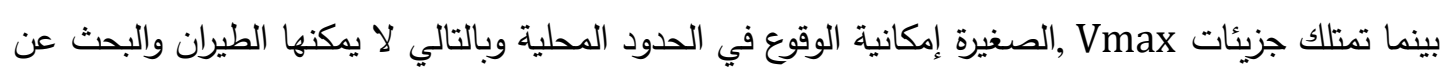
منطقة حل أفضل للبقاء في منطقة الحلنفها ، خوارزمية PSO بسيطة في المفهوم، سهلة التنفيذ ولها كفاءة

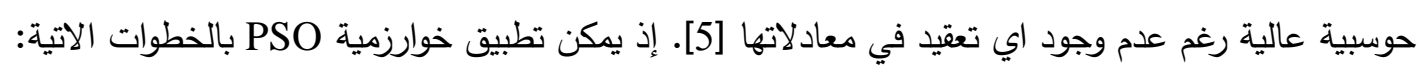

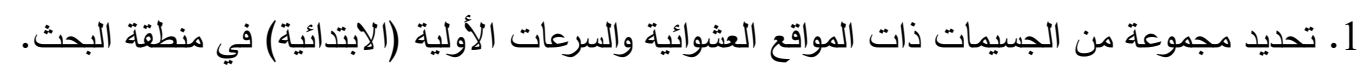

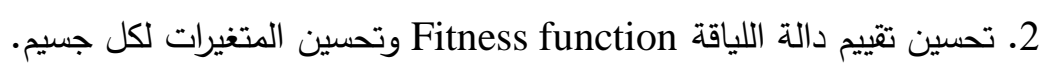

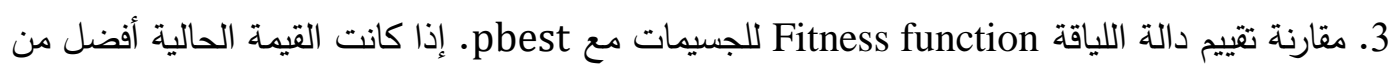

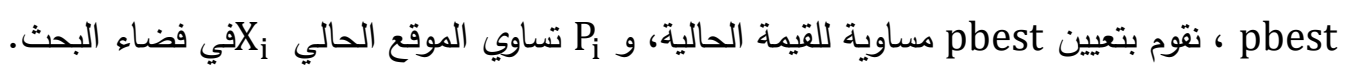

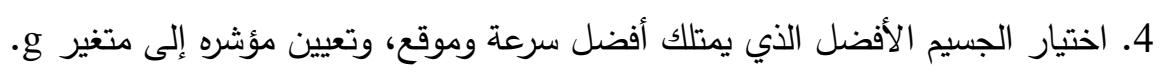

5. تغيير سرعة ومكان الجسيم وفقا" للمعادلة (1) و (2). 6. العودة إلى الخطوة (2) حتى يستوفي المعيار ، وعادة ما تكون قيمة دالة اللياقة جيدة بما فيه الكفاية أو قد تصل ولى

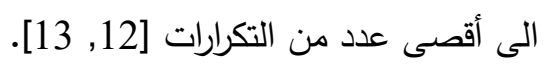

4. خوارزمية تحسين سرب الجسيمات الثنائية BPSO) Binary particle swarm optimization)

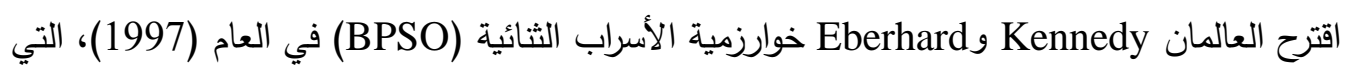
يمكن استخدامها في منطقة بحث في الفضاء المنفصل أو المتقطع. لقد صمدت خوارزمية PSO ولحل المشراب المشكلات في الفضاء المتصل Continuous search space ، وطورت خوارزمية PSO للتعامل مع المشاكل الثنائية في الفضاء المنفصل Discrete search space [14]. لتوسيع قيمة PSO الحقيقية إلى الفضاء الثنائي، فإن الجزء

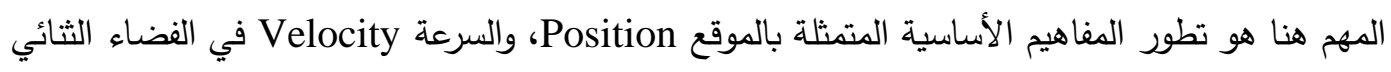

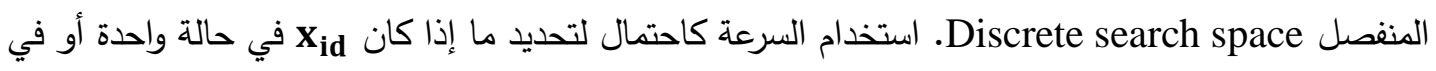

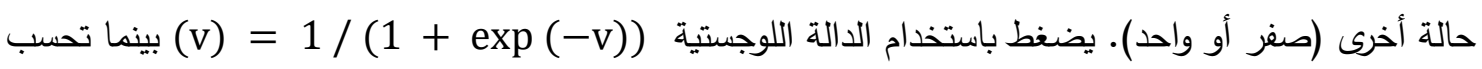

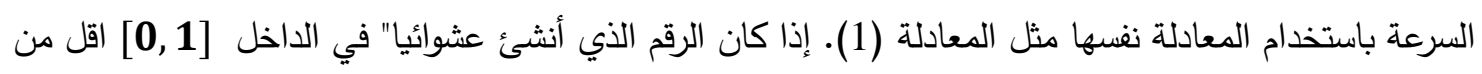

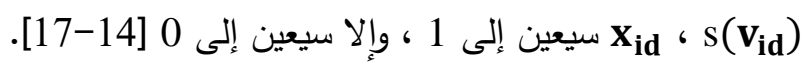




$$
\text { إذ يمكن أن نلخص خطوات خوارزمية BPSO على النحو الآتي: }
$$

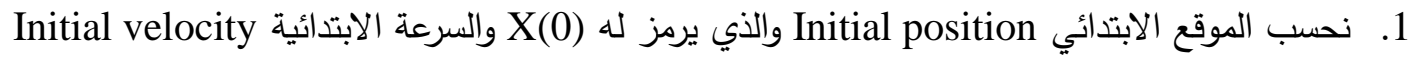
والذي يرمز لها (0) لكل جسيم Particle في السرب Swarm بكامله. 2. نقوم بوضع عداد للتكرار Iteration ونحدد عدد التكرارات المطلوبة وليكن العداد T=0 ونكرر العملية. 3. نقارن بين لياقة السرب Fitness swarm بكاملة ونأخذ أعلى لياقة Fitness. 4. نقوم بتتفيذ الخوارزمية الثنائية BPSO) Binary particle swarm optimization). 5. نحسب سرعة الجسيمات Particles velocity في كل تكرار والتعديل عليه من المعادلة (1). 6. نحدد مواقع الجسيمات Particles position في كل تكرار والتعديل عليه من المعادلة (2).

5eature selection اختيار ميزة يحدد بطريقة اختيار الميزة أهم الخصائص أو الميزات Features المستخدمة في وصف مجموعة البيانات Dataset ، إذ يكون الهدف من اختيار الميزة هو زيادة دقة التصنيف وتقليل عدد السمات المستخدمة في خوارزمية التصنيف[18]. تتكون معظم خوارزميات تحديد الميزة من ثلاثة أساليب (أجزاء) رئيسة: 1. خوارزمية البحث Search algorithm: يبحث في مجموعة فرعية من الخصائص التي تحتوي على عدد من من ملنه الميزات الأصلية. 2. وظيفة التقييم Evaluation function: تقوم هذه الوظيفة على أساس تقييم الميزات المهمة والأساسية وتميزها عن الميزات الأقل أهمية. 3. المصنف Classifier: إن الوظيفة الأساسية للمصنف بعد تقييم الميزات سوف يفصل بين الميزات الأساسية المهمة وغير المهمة [19].

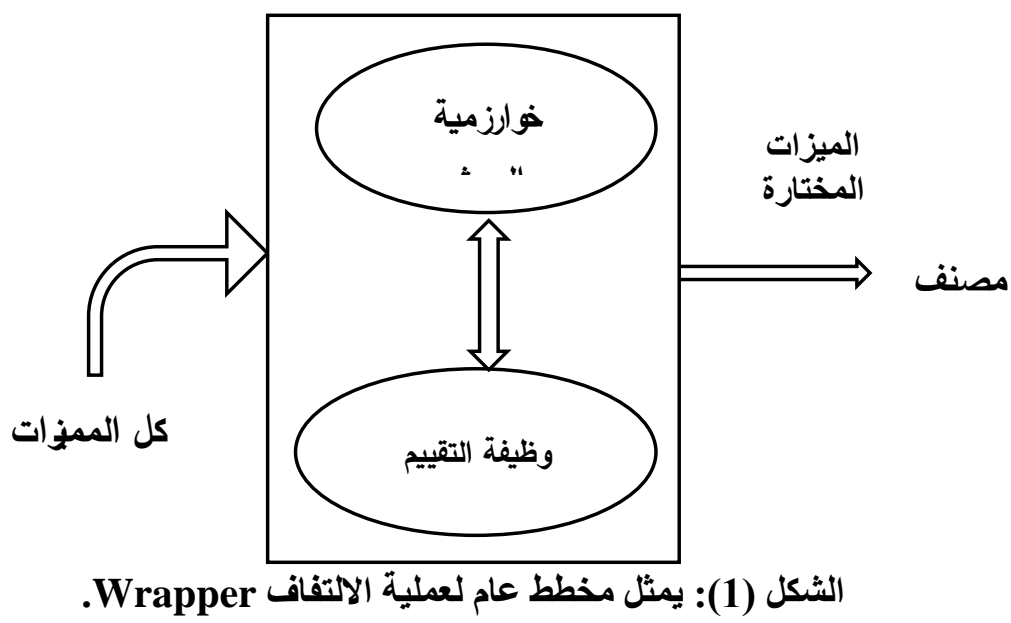

إذ تعتمد أساليب الالتفاف Wrapper وهي إحدى أقسام اختيار الميزة على تقييم جودة وكفاءة خصائص معينة من البيانات [20] بالاعتماد على دالة اللياقة Fitness function كمامبين في الشكل (1) واستتادًا إلى خوارزمية تعلم محددة (مثل خوارزمية أسراب الطيور PSO )، إذ تعتمد هذه الطريقة على خطوتين: (1) البحث عن مجموعة فرعية

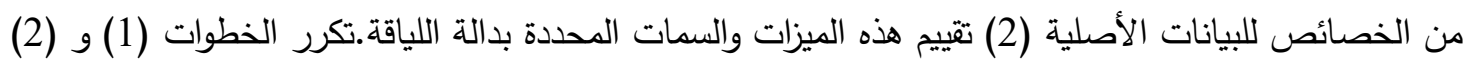
حتى يتم الوصول إلى بعض معايير التوقف التي تحدد مسبقا". 


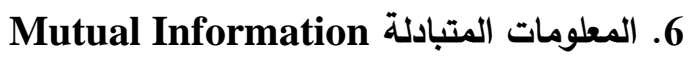

تعد تقنية المعلومات المتبادلة (MI) Mutual Information من ضمن طرائق اختيار الميزة من نوع ماته التصفية (Filtering) التي يقاس بها كم المعلومات التي يمكن الحصول عليها من مصدر معلومات معين ومقارنتها مع معلومات تم الحصول عليها من مصدر معلومات أخر وتصفيتها حسب المعلومات المشتركة بين

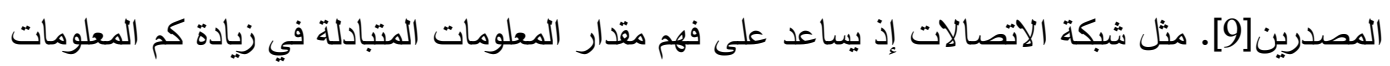

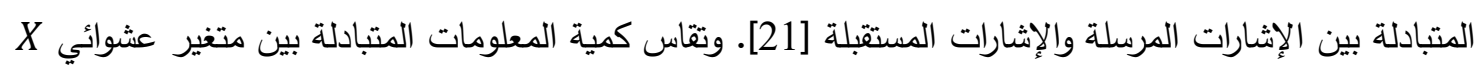
$I(X ; Y)=\sum P(X, Y) \log \frac{P(X, Y)}{P(X) P(y)}$ ومتغير عشوائي آخر Y طبقًا للمعادلة:

$=\mathrm{H}(\mathrm{X})-\mathrm{H}(\mathrm{X} \mid \mathrm{Y})$

$=\mathrm{H}(\mathrm{Y})-\mathrm{H}(\mathrm{Y} \mid \mathrm{X})$

$=\mathrm{H}(\mathrm{X})+\mathrm{H}(\mathrm{Y})-\mathrm{H}(\mathrm{X}, \mathrm{Y})$

إن طريقة المعلومات المتبادلة يمكن تطبيقها لتصفية أعداد الميزات Features في البيانات الأصلية التي يمكن ترتيبها حسب الأهمية من خلال قياس العلاقة بين ميزات معينة مع تصنيفات الفئة. هناك استخدام كلاسيكي

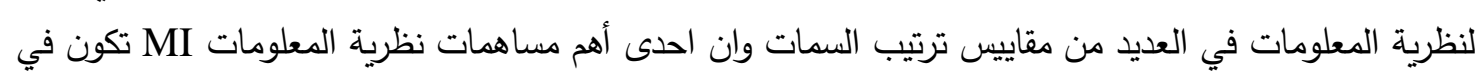

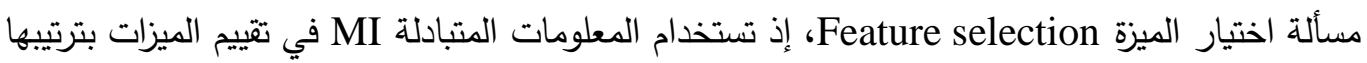
$\mathrm{I}(\mathrm{X}, \mathrm{Y})=\iint \mathrm{f}(\mathrm{x}, \mathrm{y}) \log \frac{\mathrm{f}(\mathrm{x}, \mathrm{y})}{\mathrm{f}(\mathrm{x}) \mathrm{f}(\mathrm{y})} d x d y$ تنازليا"حسب أهميتها وصياغة مجموعة الميزات طبقا" للمعادلة:

ومن المككن تمثيل المعلمات المتبادلة كما في الثكل (2)

$\mathrm{H}(\mathrm{X})$

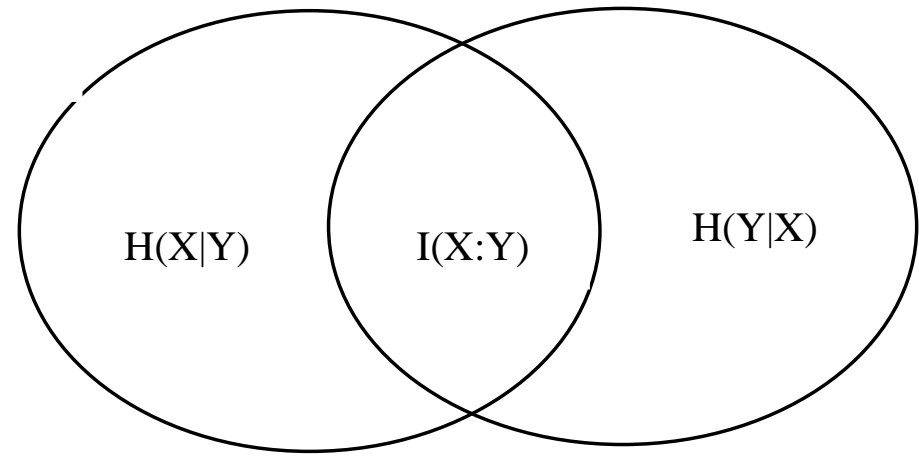

الثكل (2): تمثيل بسيط يوضح الية طريقة المعلومات المتبادلة

$\mathrm{H}(\mathrm{Y})$

أن الطريقة المستخدمة لحساب المعلومات المتبادلة هي قياس الإنتروبيا Entropy واستبدالها في شكل

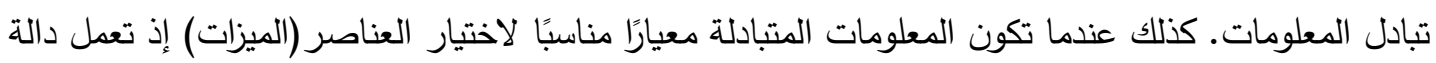
اللياقة على زيادة قيمة المعلومات المتبادلة [22]. 
7PSO_MI الخوارزمية المقترحة

تتكون الخوارزمية المقترحة في هذا البحث من مرحلتين أساسيتين، تتمثل المرحلة الأولى باستخدام طريقة

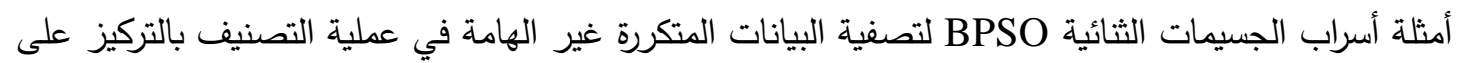
الميزات الأكثر أهمية التي يختار بالاعتماد على دالة اللياقة Fitness function (FF)، اذ يختار طبقا" للمعادلة: $F F=0.2 * C+0.8 *\left(\frac{q}{p-q}\right)$

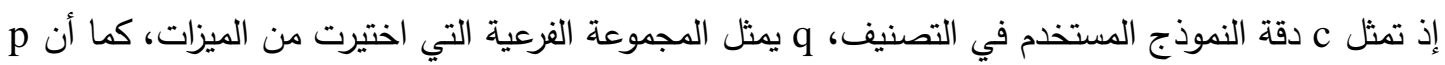
يمثل العدد الإجمالي أو الكلي للميزات الأصلية. الثكل (3) يبين عملية تحديد الميزات من خلال خوارزمية BPSO التي تتم عن طريق متجه يحتوي على سلسلة

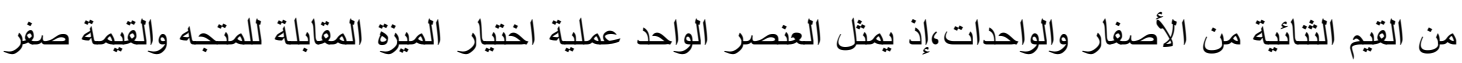
تعني ان الميزة المقابلة لها لم يتم اختيارها

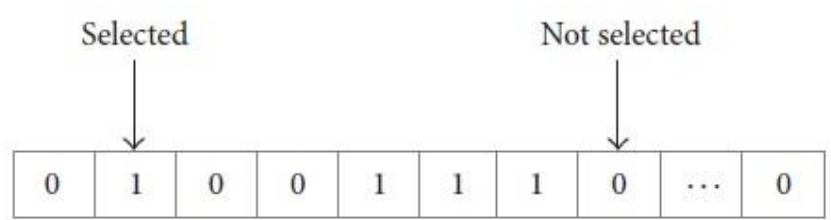

\section{الثكل (3): عينة من حل مجموعة فرعية الميزة.}

أما المرحلة الثانية من الخوارزمية المقترحة BPSO_MI فتركز على تقنية المعلومات المتبادلة MI التي يمكن اعتبارها احدى طرائق المرشح Filtering المستخدمة في اختيار الميزات، إذ يتم إدخال مجموعة الميزات المختارة من المرحلة الأولى (من خلال طريقة BPSO) الى طريقة MI ليتم ترشيحها وتخفيضها كمرحلة ثانية وفقا" لأهميتها من خلال مفهوم طريقة MI. تم اعتماد عدد قليل من الميزات في طريقة MI مقارنة بالميزات

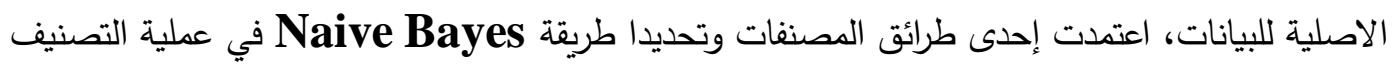

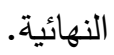

لقد قورنت الطريقة المقترحة BPSO_MI التي تعتمد عددا" اقل من الميزات Features من خلال تقنية المعلومات المتبادلة MI وذلك من خلال التطبيق على خمس مجموعات من البيانات Datasets التي تمتلك عددا"

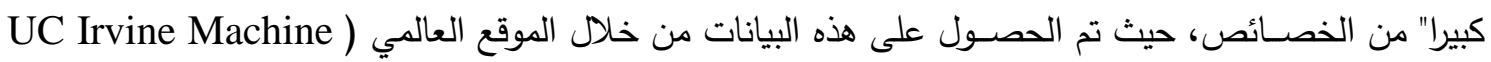

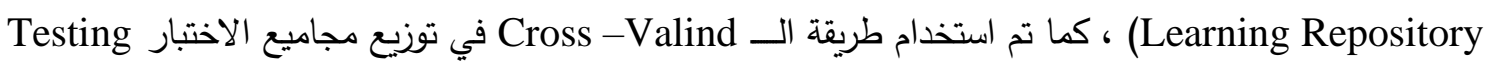

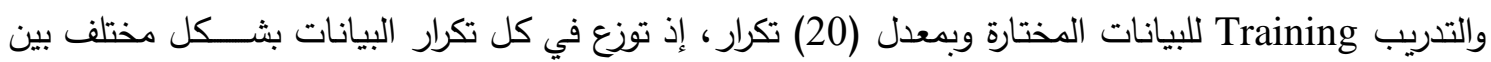
مجموعات التدريب والاختبار ليتم التأكد من مدى فعالية الخوارزمية المقترحة BPSO_MI في الحصول على فئ نتائج

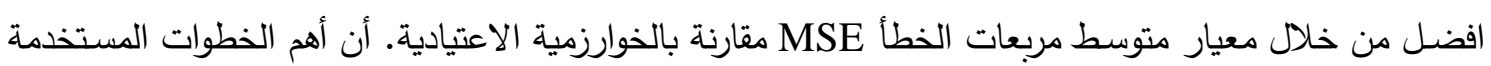
لتصنيف مجموعات البيانات باستخدام الطريقة المقترحة BPSO_MI، تكون على النحو الآتي: 1. تقيئة بيانات مصفوفة الإدخال بشكل مصفوفة X صفوفها تمثل عدد الحالات او الانماط (Patterns)

$$
\text { الكلية وأعمدتها تمثل عدد الميزات Features. }
$$

2. تستخدام طريقة ال Cross Valind لتقسيم البيانات المرضية الى مجموعات للتدريب Training وأخرى

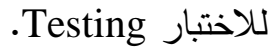




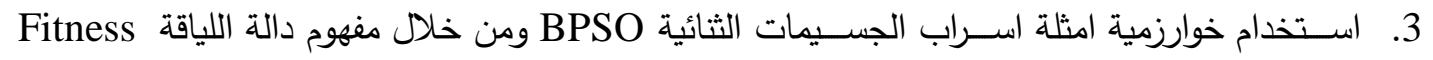
function (FF)

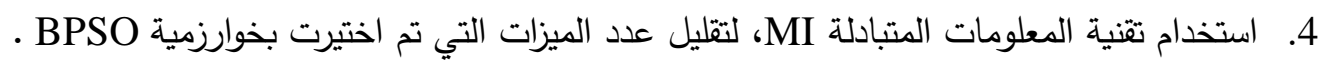
5. اختيار المصنف Naive Bayes لأجل تصنيف بيانات التدريب والاختبار.

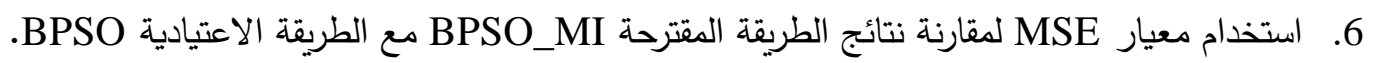

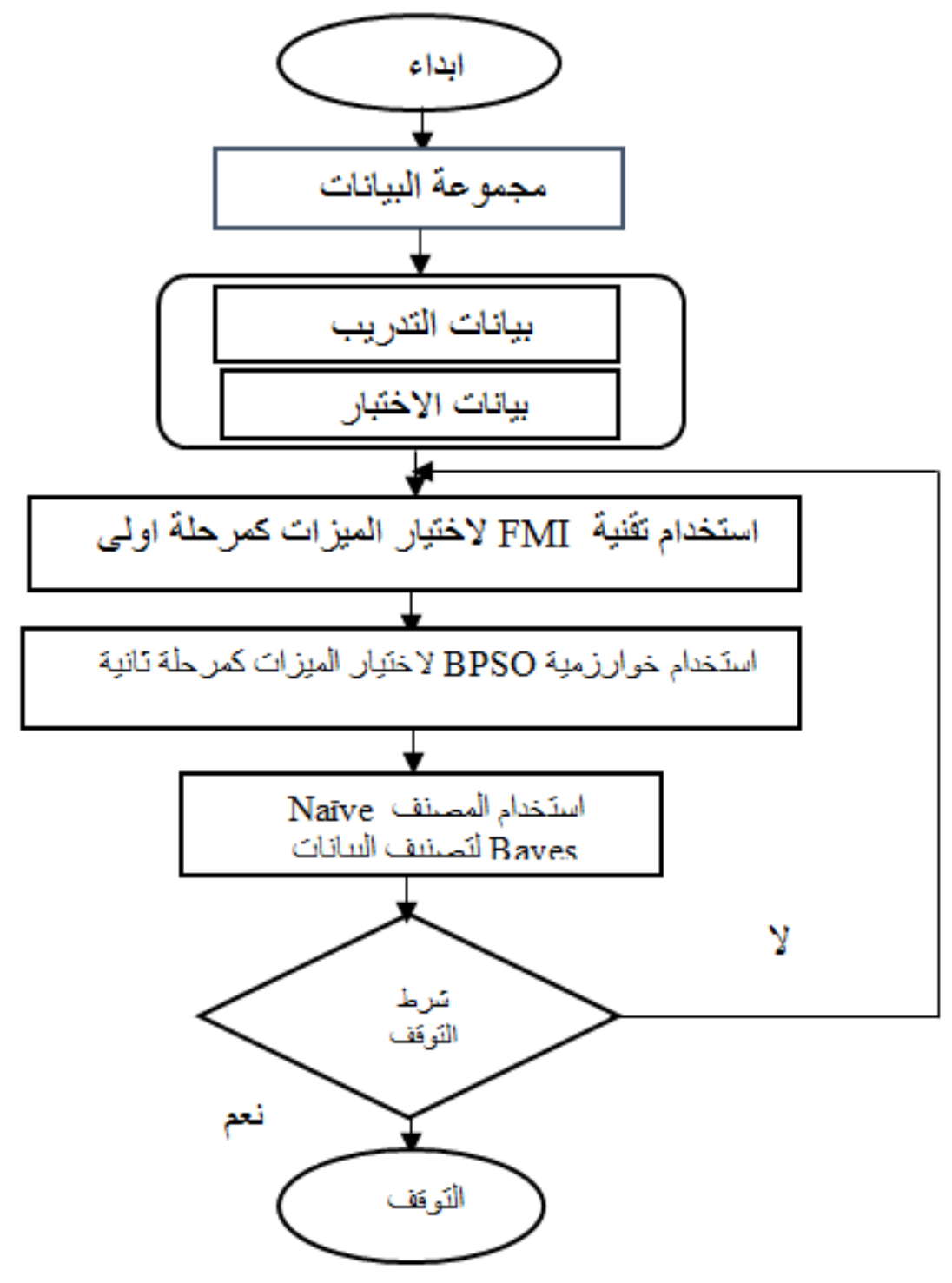

الثكل (4): مخطط انسيابي يوضح الخوارزمية المقترحة

Experimental results النتائج التجريبية طبقت الخوارزمية المقترحة BPSO_MI على خمس مجموعات من البيانات Dataset ذات الابعاد الكبيرة (او الميزات المتعددة)، والتي تم الحصول عليها من مستودع UCI العالمي، إذ تشترك جميع هذه البيانات المستخدمة في كونها ثنائية التصنيف، اذ يمكن من خلال الجدول (1) عرض وصفٍٍ لهذه البيانات المستخدمة. 
الجدول (1): يبين وصف عام لمجموعات البيانات المستخدمة.

\begin{tabular}{|c|c|c|}
\hline 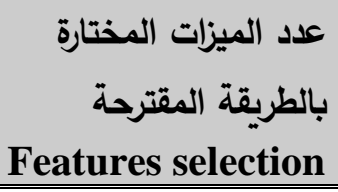 & 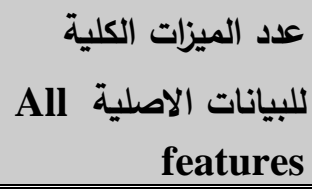 & Dataset مجموعات البيانات \\
\hline 10 & 2000 & $\overline{\text { Colon }}$ \\
\hline 3 & 15 & Japanese \\
\hline 3 & 8 & Pima \\
\hline 3 & 195 & park \\
\hline 3 & 14 & Australian \\
\hline
\end{tabular}

لقد طبقت الخوارزمية المقترحة BPSO-MI على جميع البيانات الموصوفة في الجدول (1) ومقارنتها مع الخوارزمية الاعتيادية BPSO دون اختيار ميزة، وذلك للتحقق من النتائج بشكل دقيق، وبما أن هاتين الخوارزميتين

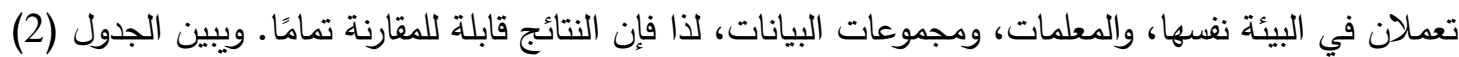
آلية هذه المقارنة على النحو الآتي:

الجدول (2): يبين مقارنة بين الخوارزمية المقترحة BPSO-MI والخوارزمية الاعتيادية BPSO من خلال معيار MSE بالنسبة لبيانات التدريب.

\begin{tabular}{|c|c|c|}
\hline الخوارزمية الاعتيادية BPSO & BI الخوارزمية المقترحة - BI & Dataset مجموعات البيانات \\
\hline $73.409 \%$ & $81.136 \%$ & Colon \\
\hline $80.559 \%$ & $82.174 \%$ & Japanese \\
\hline $75.985 \%$ & $76.487 \%$ & Pima \\
\hline $70.766 \%$ & $82.591 \%$ & park \\
\hline $80.807 \%$ & $81.863 \%$ & Australian \\
\hline
\end{tabular}

الجدول (3): يبين مقارنة بين الخوارزمية المقترحة BPSO-MI والخوارزمية الاعتيادية BPSO من خلا معيار MSE بالنسبة لبيانات الاختبار.

\begin{tabular}{|c|c|c|}
\hline الخوارزمية الاعتيادية BPSO & MI الخوارزمية المقترحة - BI & Dataset مجوعات البيانات \\
\hline $84.722 \%$ & $\% 87.222$ & Colon \\
\hline $82.222 \%$ & $\% 83.527$ & Japanese \\
\hline $77.522 \%$ & $\% 77.696$ & Pima \\
\hline
\end{tabular}




\begin{tabular}{|r|r|r|}
\hline $\mathbf{7 2 . 4 1 4 \%}$ & $\% 84.655$ & park \\
\hline $\mathbf{8 2 . 2 9 5 \%}$ & $\% 83.430$ & Australian \\
\hline \hline
\end{tabular}

نلاحظ من خلال النتائج في الجداول (1,2,3) أعلاه أن الخوارزمية المقترحة BPSO-MI أعطت ميزات اقل ونتائج أفضل من الخوارزمية الاعتيادية BPSO على جميع النيات البيانات الخمسة المستخدمة والتي أعتدات

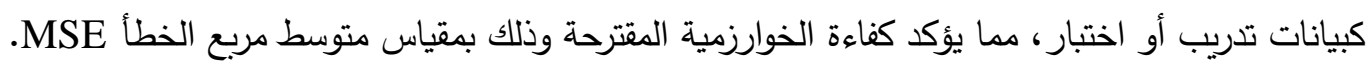

\section{Conclusions and Recommendation 9. الاستتاجات والتوصيات}

MI تضمنت هذه الدراسة اقتراح خوارزمية أمثلة أسراب الجسيمات الثنائية BPSO مع تقنية المعلومات المتبادية كطريقة لتحسين أداء التصنيف بالاعتماد على مجموعات فرعية من الميزات Features المهمة لمجموعة البيانات.

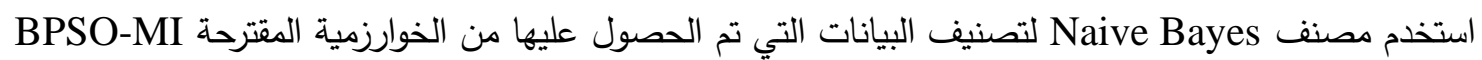

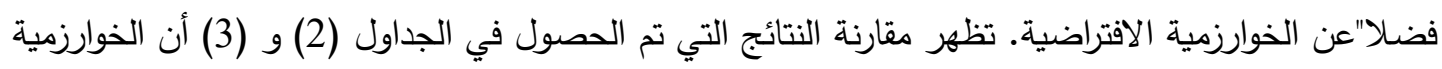

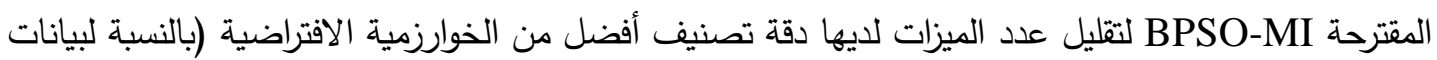
الاختبار والتتريب)، أزيلت الميزات غير الضرورية للطريقة المقترحة BPSO_MI من خلادل تلال مرحلتين وبالتالي تحسين نتائج التصنيف الثشاملة.

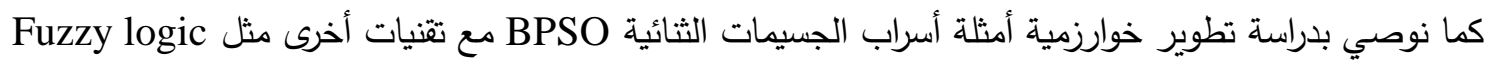
أو خوارزميات ذكاء السرب Intelligence Swarm من خلال إيجاد أفضل الميزات Features المستخدمة

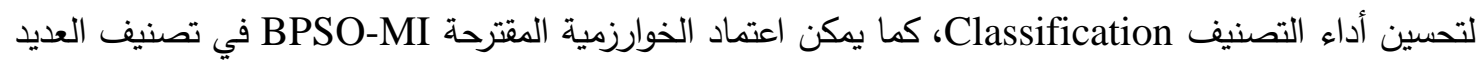
من البيانات ذات الابعاد الكبيرة مثل الجينية وغيرها. 


\section{المصادر}

[1] T. S. Babu, J. P. Ram, T. Dragičević, M. Miyatake, F. Blaabjerg, and N. Rajasekar, "Particle swarm optimization based solar PV array reconfiguration of the maximum power extraction under partial shading conditions," IEEE Transactions on Sustainable Energy, vol. 9, pp. 74-85, 2018.

[2] S. Cheng, H. Lu, X. Lei, and Y. Shi, "A quarter century of particle swarm optimization," Complex \& Intelligent Systems, 2018.

[3] S. Dutta and S. Singh, "Optimal rescheduling of generators for congestion management based on particle swarm optimization," IEEE Transactions on Power Systems, vol. 23, pp. 1560-1569, 2008.

[4] R. Eberhart and J. Kennedy, "A new optimizer using particle swarm theory," in Micro Machine and Human Science, 1995. MHS'95., Proceedings of the Sixth International Symposium on, 1995, pp. 39-43.

[5] S. Rafi, A. Kumar, and G. K. Singh, "An improved particle swarm optimization method for multirate filter bank design," Journal of the Franklin Institute, vol. 350, pp. 757-769, 2013.

[6] M. Abdul-Razek, "Detection of Heart Disease using Binary Particle Swarm Optimization," Damietta University Publication, vol. 1, 2014.

[7] P. Ghamisi and J. A. Benediktsson, "Feature selection based on hybridization of genetic algorithm and particle swarm optimization," IEEE Geoscience and Remote Sensing Letters, vol. 12, pp. 309-313, 2015.

[8] M. Mafarja and S. Mirjalili, "Whale optimization approaches for wrapper feature selection," Applied Soft Computing, vol. 62, pp. 441-453, 2018.

[9] E. Hancer, B. Xue, and M. Zhang, "Differential evolution for filter feature selection based on information theory and feature ranking," Knowledge-Based Systems, vol. 140, pp. 103-119, 2018.

[10] A. Rezaee Jordehi, "Enhanced leader particle swarm optimisation (ELPSO): An efficient algorithm for parameter estimation of photovoltaic (PV) cells and modules," Solar Energy, vol. 159, pp. 78-87, 2018.

[11] Z. Wu, W. Gu, R. Wang, X. Yuan, and W. Liu, "Economic optimal schedule of CHP microgrid system using chance constrained programming and particle swarm optimization," in Power and Energy Society General Meeting, 2011 IEEE, 2011, pp. 1-11. 
[12] Y. Shi and R. C. Eberhart, "Empirical study of particle swarm optimization," in Evolutionary computation, 1999. CEC 99. Proceedings of the 1999 congress on, 1999, pp. 1945-1950.

[13] R. Thangaraj, M. Pant, A. Abraham, and P. Bouvry, "Particle swarm optimization: hybridization perspectives and experimental illustrations," Applied Mathematics and Computation, vol. 217, pp. 5208-5226, 2011.

[14] M. S. Mohamad ,S. Omatu, S. Deris, M. Yoshioka, A. Abdullah, and Z. Ibrahim, "An enhancement of binary particle swarm optimization for gene selection in classifying cancer classes," Algorithms for Molecular Biology, vol. 8, p. 15, 2013.

[15] M. N. Elbedwehy, H. M. Zawbaa, N. Ghali, and A. E. Hassanien, "Detection of heart disease using binary particle swarm optimization," in Computer Science and Information Systems (FedCSIS), 2012 Federated Conference on, 2012, pp. 177182.

[16] A. H. El-Maleh, A. T. Sheikh, and S. M .Sait, "Binary particle swarm optimization (BPSO) based state assignment for area minimization of sequential circuits," Applied Soft Computing, vol. 13, pp. 4832-4840, 2013.

[17] I. Jain, V. K. Jain, and R. Jain, "Correlation feature selection based improvedBinary Particle Swarm Optimization for gene selection and cancer classification," Applied Soft Computing, vol. 62, pp. 203-215, 2018.

[18] Y. Zhang, D. Gong, Y. Hu, and W. Zhang, "Feature selection algorithm based on bare bones particle swarm optimization," Neurocomputing, vol. 148, pp. 150-157, 2015.

[19] M. Zaffar, K. Savita, M. A. Hashmani, and S. S. H. Rizvi, "A Study of Feature Selection Algorithms for Predicting Students Academic Performance," INTERNATIONAL JOURNAL OF ADVANCED COMPUTER SCIENCE AND APPLICATIONS, vol. 9, pp. 541-549, 2018.

[20] B. Liu, E. Blasch, Y. Chen, D. Shen, and G. Chen, "Scalable sentiment classification for big data analysis using naive bayes classifier," in Big Data, 2013 IEEE International Conference on, 2013, pp. 99-104.

[21] Y. Prasad, K. K. Biswas, and M. Hanmandlu, "A recursive PSO scheme for gene selection in microarray data," Applied Soft Computing, vol. 71, pp. 213-225, 2018.

[22] M. A. Sulaiman and J. Labadin, "Feature selection based on mutual information," in IT in Asia (CITA), 2015 9th International Conference on, 2015, pp. 1-6. 